\title{
Correlated Atomic Force Microscopy and Single Molecule Localization Microscopy
}

Pascal D. Odermatt ${ }^{1}$, Arun Shivanandan ${ }^{2}$, Hendrik Deschout ${ }^{2}$, Radek Jankele ${ }^{2}$, Adrian, P. Nievergelt ${ }^{1}$, Lely Feletti ${ }^{2}$, Michael W. Davidson ${ }^{3}$, Aleksandra Radenovic ${ }^{2}$ and Georg E. Fantner ${ }^{1}$

${ }^{1}$ Laboratory for Bio- and Nano-Instrumentation, Institute of Bioengineering, School of Engineering, EPFL, 1015 Lausanne, Switzerland

${ }^{2}$ Laboratory of Nanoscale Biology, Institute of Bioengineering, School of Engineering, EPFL, 1015 Lausanne, Switzerland

${ }^{3}$ National High Magnetic Field Laboratory, Florida State University, Tallahassee, Florida, USA. Department of Biological Science, Florida State University, Tallahassee, Florida, USA.

Nanoscale characterization of living samples has become essential for modern biology. Atomic Force Microscopy (AFM) creates topological images of fragile biological structures from biomolecules to living cells in aqueous environment [1]. However, correlating nanoscale structure to biological function of specific proteins can be challenging. Fluorescence microscopy on the other hand is capable of revealing specific biochemical structures with great specificity. The combination of fluorescence microscopy and atomic force microscopy has therefore long since been a very powerful tool. However, the resolution of the two techniques differs vastly, which has made correlated imaging somewhat ambiguous. With the rapid development of single molecule localization microscopy techniques [2] (SMLM), such as PALM (photo activated localization microscopy) and STORM (stochastic optical reconstruction microscopy), this gap in resolution is closing. These methods are therefore often called "super resolution" microscopy techniques. Correlated AFM/SMLM therefore hold tremendous potential for structural and cellular biology. To make full use of a combination of the two techniques it is essential that each technique retains its optimal performance, even in combination experiments. In this work we present a combination AFM/SMLM instrument that allows each of the two techniques to operate at its full potential. The special construction of the microscope allows for integrating an AFM with an optical microscope without an increase in vibrational noise. The AFM noise performance on the combined system was measured to be 0.24 Angstrom, which is equivalent to that of very low noise stand alone AFMs. The resolution in the SMLM image obtained with the combined system shows a mean localization precision of $12.5 \mathrm{~nm}$, which is also equivalent to that of dedicated systems.

AFM offers the unique capability of observing living cells in physiological solution with exceptional surface resolution, but internal cellular structures remain hidden. SMLM can reveal structures on the inside of cells. This is particularly interesting for microtubules or actin filaments, which play a big role in cell stability and intra-cellular transport. Actin filaments have been studied extensively both with atomic force microscopy as well as super-resolution optical microscopy. To compare the imaging resolution of atomic force microscopy and super resolution optical microscopy we used direct Stochastic Optical Reconstruction Microscopy (dSTORM)/AFM, to directly correlate and quantify the density of localizations using both imaging modalities along (F-) actin cytoskeletal filaments. After having established the correlation of the two techniques by measuring the same aspect of the same sample, we demonstrate the ability of measuring complementary aspects of a sample. Photo Activated Light Microscopy (PALM)/AFM, we provide correlative images of bacterial and mammalian cells in aqueous condition. The complementary information provided by the two techniques opens a new dimension for structural and functional nanoscale biology. [3] 


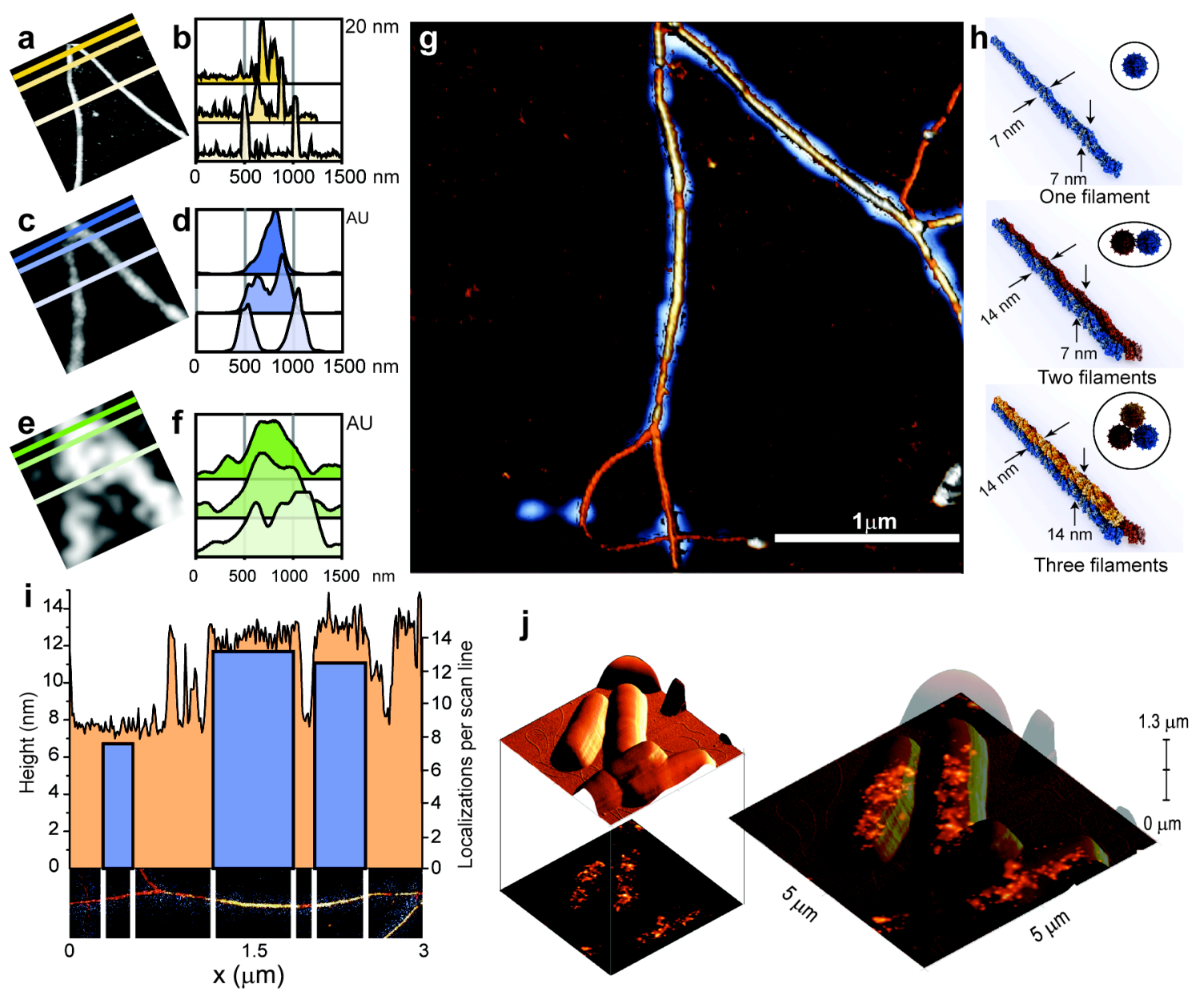

Figure 1: Comparison of AFM, dSTORM and TIRF imaging resolution from correlative imaging. ( a, c \& e) AFM, dSTORM and TIRF images respectively $(1.5 \mu \mathrm{m} \times 1.5 \mu \mathrm{m})$ of correlated filaments. Lines and colours indicate the location of profiles shown in $(\mathrm{b}, \mathrm{d} \& \mathrm{f})$. ( $\mathrm{g}$ ) Overlay of dSTORM probability map (blue) and 3D rendered AFM image (yellow-brown-). (h) Different F-actin arrangements suggested based on the AFM data. (i) Bar plot showing the number of localizations detected at a specific height measured by AFM divided by the number of pixels having that particular height. Bin size: $2 \mathrm{~nm}$. Orange: Maximum height projection of the AFM section showed below the plot. Blue: Number of localizations detected in regions between the white lines per line scan of the AFM. (j) Correlative AFM/PALM image of E.coli bacteria expressing RNA polymerase-mEos2.

[1] Henderson, E., Haydon, P. G., Sakaguchi, D. S. Science 257, (1992), p5078, 3.

[2] Betzig, E., et al. Science 313 (2006), p. 1642-1645

[3] This work was financially supported by FNS grants No. 200021-125319, No. 20021-132206, No. 205321-134786 and No. 205320-152675 as well as the European Union's Seventh Framework Program FP7/2007-2011 under grant agreement 286146 and FP7/2007-2013/ERC grant agreement 307338. A. Shivanandan was funded by a PhD fellowship grant from NCCBI. We thank Dr. P. Annibale for initial help with the instrument. We thank Lina Carlini for discussion and Prof. Mike Heilemann on the generous gift of bacterial strain expressing mEos2-RNAP 